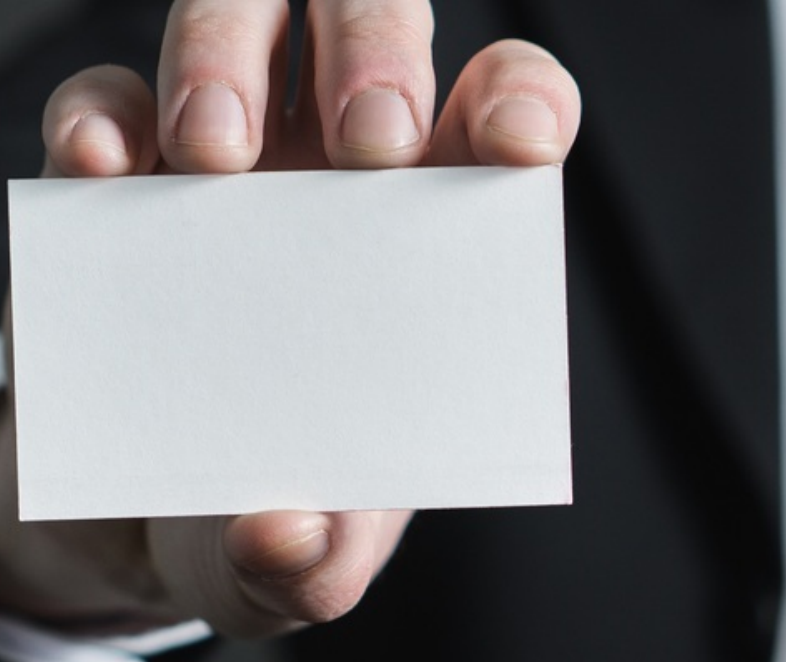

\title{
Charles Taylor: Una Aproximación a la Noción de Reconocimento e Identidad -Charles Taylor: An Approach to the Notion of Recognition and Identity
}

\author{
Authors: Luis Enrique Silva Vieira \\ Submitted: $\quad 25$. October 2020 \\ Published: $\quad$ 26. October 2020 \\ Volume: 7 \\ Issue: 5 \\ Affiliation: Pontificia Universidad Católica Santa Rosa UCSAR, Caracas, \\ Venezuela \\ Languages: Spanish, Castilian \\ Keywords: Identity, Recognition, Hegel, Charles Taylor \\ Categories: Humanities, Social Sciences and Law, Life Sciences \\ DOI: $\quad$ 10.17160/josha.7.5.715
}

\section{Abstract:}

The thought of identity and recognition occupies an increasingly important place in Charles Taylor's thinking. It has even sparked the interest of thinkers like Michel Walzer and Jürgen Habermas to name just a few. In this way, his critique of the innumerable problems of the present ended up adapting a historical form. This article analyzes the diverse forms of approach to thematic creation in the work Philosophical Arguments, concentrating exclusively on Chapter 12 "The politics of recognition".

\section{JOSHA Joumano ossanere Humanities and Arts}




\section{Charles Taylor: Una Aproximación a la Noción de Reconocimento e Identidad - Charles Taylor: An Approach to the Notion of Recognition and Identity}

\section{Luis Enrique Silva Viera}

Afiliación: Pontificia Universidad Católica Santa Rosa UCSAR. Caracas, Venezuela.

\section{RESUMEN}

La idea de identidad y reconocimiento ocupa en el pensamiento de Charles Taylor un lugar cada vez más importante. Incluso ha despertado el interés de pensadores como Michel Walzer y Jürgen Habermas por nombrar sólo algunos. De esta manera, su crítica a los innumerables problemas del presente terminó adaptando una forma histórica. En este artículo se analizan las formas diversas de aproximación a la creación tematizable en la obra "Argumentos filosóficos" Philosophycal Arguments concentrándonos exclusivamente en el Capítulo 12 "La política del reconocimiento". Se pone de manifiesto en primer lugar, la importancia del pensamiento hegeliano en Taylor y su influencia en su pensamiento, a saber, en segundo lugar, la inquietud del pensador canadiense sobre los problemas de identidad y reconocimiento que le inquieta dentro de la sociedad circundante, por tanto, el reflexionar y comunicar acerca de la relevancia de las acciones humanas es, sin lugar a dudas, el telos que se propone Taylor.

Keywords: Identity, Recognition, Hegel, Charles Taylor

Palabras claves: Identidad, Reconocimiento, Hegel, Charles Taylor 


\begin{abstract}
The thought of identity and recognition occupies an increasingly important place in Charles Taylor thinking. It has even sparked the interest of thinkers like Michel Walzer and Jürgen Habermas to name just a few. In this way, his critique of the innumerable problems of the present ended up adapting a historical form. This article analyzes the diverse forms of approach to thematic creation in the work Philosophycal Arguments, concentrating exclusively on Chapter 12 "The politics of recognition". First, the importance of Hegelian thought in Taylor and its influence on his thought is shown, namely, secondly, the Canadian thinker's concern about the problems of identity and recognition that worries him within the surrounding society, therefore, reflecting and communicating about the relevance of human actions is, without a doubt, the telos that Taylor proposes.
\end{abstract}

Keywords: Identity, Recognition, Hegel, Charles Taylor. 
Pero esta naturaleza universal independiente, en la que la negación es como negación absoluta, es el género como tal o como autoconciencia. La autoconciencia sólo alcanza su satisfacción en otra autoconciencia. ${ }^{l}$

Georg Wilhelm Friedrich Hegel

En diversos aspectos la política contemporánea gira en torno de la necesidad, a veces de la exigencia, de reconocimiento. ${ }^{2}$

Charles Taylor

El objetivo central de nuestra indagación es analizar cuidadosamente la noción propuesta por Charles Taylor con respecto a Reconocimiento e Identidad, para tal fin nos proponemos revisar en primer lugar los antecedentes que dieron lugar a dicha noción, en segundo lugar examinaremos su texto "Argumentos Filosóficos" esencialmente el capítulo que hace referencia a "La política del Reconocimiento" entre otros que consideremos para la investigación.

Sin dudas Taylor es un gran conocedor del filósofo alemán Georg Wilhelm Friedrich Hegel (1770-1831), en nuestra opinión su dominio sobre este autor lo encontramos en su excelente texto Hegel (1975). Dentro de este marco consideramos que la idea de reconocimiento fue tomada por nuestro autor a partir del Capítulo IV de la Fenomenología del Espíritu del pensador de Stuttgart y que hace referencia a La verdad de la Certeza de sí Mismo, en tal sentido el pensador canadiense nos dice que "Nos viene a la memoria Hegel, con su célebre dialéctica del amo y del esclavo"3 por tanto puede argüirse que la idea fundamental que nos va a permitir conocer a profundidad la noción que tiene Hegel sobre reconocimiento es a partir de una revisión de dicho texto, finalmente dedicaremos en lo que sigue cómo Taylor toma esta idea y la desarrolla introduciendo argumentos que le dará forma, sustento a su indagación.

Hablaremos igualmente sobre la idea de identidad que tiene fundamentalmente Taylor en el texto que trabajaremos, asimismo debemos recordar que los conceptos reconocimiento e

\footnotetext{
${ }^{1}$ Hegel, G.W.F., Fenomenología del Espíritu, México, Editorial Fondo de Cultura Económica, Decimosexta edición, 2006, p. 112.

2 Taylor, Ch., Argumentos filosóficos, Barcelona, Editorial Paidós, 2012, p. 293.

${ }^{3}$ Ibid., p. 294.
} 
identidad están articulados, están imbricados y por consiguiente no pueden ir separados pues no tendrían sentido.

\section{1-. LA IDEA DE RECONOCIMIENTO EN HEGEL}

La necesidad de los hombres de ser reconocidos estriba fundamentalmente en formar parte y en pertenecer a una determinada sociedad, la cual los hombres forman parte inmanente “...el tema del reconocimiento -nos dice Taylor- es tratado por primera vez por Hegel de una manera que ha ejercido gran influencia."4 Hegel en su Fenomenología del Espíritu explica magistralmente cómo ha de darse ese movimiento dialéctico y por tanto esencial en su pensamiento. Hemos recogido en la lectura de Francis Fukuyama la interpretación que tiene él pero siempre a la luz del pensamiento de Alexandre Kojève ${ }^{5}$ del capítulo IV "La verdad de la Certeza de sí Mismo" en la Fenomenología del Espíritu.

Consecuentemente analizaremos puntualmente aquí lo que nos interesa para descifrar lo que Taylor nos quiere decir en su Philosophycal Arguments a la luz del pensamiento hegeliano y observaremos diferencias y coincidencias mediante el análisis.

La Fenomenología del Espíritu del pensador de Stuttgart es, sin duda, una obra difícil, compleja de abordar en tal sentido para lograr entenderla el lector debe estar familiarizado con los conceptos y categorías que utiliza el autor, no nos detendremos aquí en un estudio minucioso del pensamiento hegeliano, más bien intentaremos explicar el momento dialéctico mediante el cual la autoconciencia solo se satisface con otra autoconciencia, observar las ideas fundamentales que dieron pie a la interpretación que hace Taylor sobre reconocimiento e identidad.

En primer lugar hablaremos de ese solipsismo que tanto manifestó Johann Gottlieb Fichte (1762-1814). Para este filósofo alemán era fundamental la idea de un Yo = Yo absoluto es decir, todo es idéntico así mismo y de ahí no trascendía, "El fundamento del sistema de Fichte - nos dice Hegel- es la intuición intelectual, pensar puro de sí mismo, autoconciencia

\footnotetext{
${ }^{4}$ Taylor, Ch., Argumentos filosóficos, cit., p. 302.

${ }^{5}$ Alexandre Kojève, tiene un texto con el título "La Dialéctica del Amo y del Esclavo en Hegel" publicado en español por la Editorial Pléyade, Buenos Aires y traducido por Juan José Sebreli. (1971) consideramos sin embargo que el texto nos presenta una interpretación del pensamiento de Hegel digno de ser revisado, cosa que no es el problema que nos trae, nos toca por tanto ser lo más preciso y ajustado al pensamiento de Hegel, en tal sentido citaremos otras fuentes incluyendo la Fenomenología del Espíritu de Hegel.
} 
pura, yo = yo, yo soy; lo absoluto es sujeto-objeto, y el yo es esta identidad de sujeto y objeto"6 ahora bien, esta cita nos parece fundamental para entender la idea de solipsismo en tanto subjetivismo extremo, en realidad Fichte se está refiriendo a la noción de lo absoluto sujeto-objeto pero como uno es decir, no escindido, lo cual es criticado por Hegel por su inconsistencia dentro del pensar.

Con respecto a esto último el filósofo de Stuttgart estaba de acuerdo, sin embargo él pensaba que estaba incompleta la idea, pues para nuestro autor lo esencial es que se dé esta forma: $Y_{0}=Y_{o}=$ No-Yo, es decir todo es igual así mismo, sin embargo hay algo fuera de mí que es un No-Yo que hace que me dé cuenta quién soy, por ejemplo: sé que soy Luis pero para saber que soy Luis debe haber fuera de mi uno o unos NO luises es decir Juan, Pedro, María, etc., que me permitan saber quién soy.

Es necesario recalcar que lo que quiere expresar Hegel con la identidad de la identidad y No identidad, es hablar de la identidad en la diferencia, él lo diría más o menos así, sé que soy yo porque existe un yo diferente a mí que está fuera $\mathrm{YO}=\mathrm{YO}$ y NO-YO. Asimismo el filósofo alemán había asomado germinalmente el problema de la oposición en su Fragmento de Sistema allí nos dice que, "precisamente la vida no se puede considerar sólo en cuanto unificación, en cuanto relación: tiene que ser considerada simultáneamente como oposición"7 precisamente es en ese sentido que debemos entender el reconocimiento, nos reconocemos a nosotros mismos en el otro en tanto que nosotros.

Observamos enseguida que la noción de identidad coincide con la idea que tiene Taylor de ella pero con algunos matices que saltan a la vista, Hegel según Eduardo Vásquez piensa la identidad en estos términos "No es que Hegel niegue la identidad. Lo que afirma es que la identidad tiene que ser negada o (mediada) tiene que entrar en relación con lo otro, con lo que ella no es, pero sin lo cual no podría ser." ${ }^{8}$ Efectivamente, sólo entrando en relación con lo otro existe la identidad por tanto, se debe reconocer lo otro en tanto que es mi negación.

\footnotetext{
${ }^{6}$ Hegel, G.W.F., Diferencia entre los sistemas de filosofía de Fichte y Schelling. Madrid, Editorial-Tecnos, S.A., 1990, p. 57.

${ }^{7}$ Hegel, G.W.F., Escritos de Juventud. (3ra ed.) México, Editorial Fondo de Cultura Económica, 1998, p. 401.

${ }^{8}$ Vásquez, E., Hegel un desconocido. Universidad de los Andes, Mérida, Consejo de publicaciones. 1998, p. 174.
} 
Hablemos brevemente de la autoconciencia y sus implicaciones con la idea de reconocimiento e identidad que nos habla Taylor. Volvamos nuevamente a Fukuyama que haciendo una lectura interpretativa de Hegel pero siempre a la luz del pensamiento de Kojève a quien se refiere cariñosamente como (Hegel-Kojève) intenta explicar cómo esa idea de reconocimiento encaja en una sociedad como la nuestra, de este modo Fukuyama apoyándose en Hegel-Kojève nos dice que "La segunda razón para volver a Hegel es que su concepción de la historia como una $<<$ lucha por el reconocimiento $>>$ es un modo muy útil e iluminador de ver el mundo contemporáneo."9 Es una interpretación acertada si observamos las coincidencias de sus argumentos con la de Taylor que revisaremos en la segunda parte de esta indagación.

Efectivamente, Fukuyama se sirve de la dialéctica del amo y el esclavo para fundamentar la idea que él tiene de reconocimiento, sin embargo nos serviremos aquí puntualmente de lo que nos concierne. Cuando nosotros nos referimos a Autoconciencia y vida, la autoconciencia no se conforma con el saber del objeto, en otras palabras, la autoconciencia necesita ser en sí y para sí, la autoconciencia sólo estará satisfecha en cuanto es para otra autoconciencia ¿Qué quiere decir esto? Se trata de que quiere ser reconocida por otra autoconciencia, en eso consiste la Vida porque estando cada autoconciencia cierta de sí misma ésta demanda ser reconocida por otra autoconciencia es allí donde comienza el conflicto donde cada una de estas autoconciencias aborrecen su vida y también la de otra autoconciencia, sin embargo esta experiencia, -y esto es bien importante- esta lucha entre las autoconciencias, le es esencial la vida ya que sin la vida esta no puede ser negada, Palmier nos dice que "La conciencia de sí que es deseo no podrá descubrir la verdad más que en otra conciencia, que sea tan viviente como ella. Sólo cuando encuentra en otra conciencia un eco de su propio eco puede encontrar en ella su verdad" 10 a propósito de la dialéctica del amo y el esclavo Marx luego logrará con su extraordinario genio desarrollar la lucha de clases donde piensa él está el motor de la historia.

Por otra parte, el Deseo que está enfrente forma parte de lo viviente que vive solo para desear, al respecto nos dice Nancy que "Temblando con el temblor del otro, el sí-mismo llega en el deseo. La conciencia de sí es esencialmente deseo, porque es conciencia de sí en cuanto

\footnotetext{
${ }^{9}$ Fukuyama, F., El fin de la historia y el último hombre. Barcelona, Editorial Planeta, S.A., 1992, p. 209.

${ }^{10}$ Palmier, J., Hegel. (4a ed.) México, Editorial Fondo de Cultura Económica, D.F. México, 2006, p. 43.
} 
que y a partir de su conciencia de lo otro." ${ }^{11}$ Lo verdadero es alcanzado por lo deseado, que ha recorrido la conciencia para llegar al sí mismo y continuamente va engendrando cada vez más deseos, aquí Heidegger tiene algo que decirnos y apunta lo siguiente:

El hacía-sí, el cual pertenece al ser-en-el-interior-de-sí-mismo, el retorno a-sí como verdad, es aprehendido como deseo, como el afán del sí-mismo por él mismo, [...] la satisfacción corre por la senda de la conciencia de los objetos y por eso alcanza su meta, sino que engendra cada vez nuevos deseos. ${ }^{12}$

Eso quiere decir que ¿Hegel se está refiriendo más a que el deseo no es un deseo de un objeto como tal, que nos es dado? no. Es el deseo de una conciencia igual a la nuestra "El deseo es deseo del sujeto, y no hay objeto del deseo"13 comienza entonces el problema o más bien la guerra del reconocimiento por el otro que se da en la "Dialéctica del amo y el esclavo." Es importante considerar aquí que en un principio el hombre es deseoso de los objetos que le rodean pero no tardara en darse cuenta como acertadamente nos dice Marcuse que "llega a sentir que los objetos no son el fin verdadero de su deseo, y que sus necesidades sólo pueden satisfacerse a través de la asociación con otros individuos."14

La vida se va generando desde dentro de sí, "La unidad es - nos comenta Heidegger$<<$ la quietud de sí misma como infinitud absolutamente inquieta $>>$. El ser es aprehendido como autonomía, el constante ser-sí-mismo que se mantiene en el interior de sí" ${ }^{15}$ volviendo a Hegel en sus escritos de juventud hacía referencia a La Vida pero no aún en los términos de la Fenomenología del Espíritu, en su juventud pensaba más en religión, en una concepción teológica más que en filosofía, sin embargo en sus pensamientos juveniles comenzaba a germinar en él lo que más tarde sería todo su sistema filosófico prueba de ello es su Fragmento de Sistema de Frankfurt referencia indiscutible donde ya comienza asomar el problema de La Vida pero como tema teológico, dice Hegel que "Si el hombre pone simultáneamente la vida infinita como espíritu del otro fuera de sí (puesto que ahora está limitado) y así mismo fuera de sí, fuera de lo limitado, elevándose hacia lo viviente y

\footnotetext{
${ }^{11}$ Nancy, J., Hegel. La inquietud de lo negativo. Madrid, Arena Libros, S.L., 2005, p. 68

12 Heidegger, M., La Fenomenología del Espíritu de Hegel, Madrid, Editorial Alianza, S.A., 1995, p. 198.

${ }^{13}$ Nancy, J., Hegel. La inquietud de lo negativo..., cit., p. 69.

${ }^{14}$ Marcuse, H., Razón y Revolución. (3a ed.) Madrid, Alianza Editorial, S.A., 1996, p. 116.

${ }^{15}$ Heidegger, M., La Fenomenología del Espíritu..., cit., p. 205.
} 
unificándose íntimamente con él, entonces adora a Dios"16 esta idea por supuesto es reformulada en el devenir de su vida transformándose en una concreción en su pensamiento maduro.

Por su parte Taylor en su texto Hegel (1975) nos dice que "la integridad que Hegel postula como la meta es la negación de la dependencia de algo más, es el reconocimiento del yo en todo lo que me es esencial." 17 Así Taylor configura de entrada lo que más adelante con su idea de reconocimiento dará a su indagación sobre este tema.

El pensamiento hegeliano más trabajado en sus escritos posteriores está evidentemente en la Fenomenología del Espíritu que plantea la vida en otros términos se refiere el pensador alemán específicamente no a la vida en general sino a la vida del ser humano como tal, comienza a observar que debe abandonar el pensamiento que lo ha llevado a la autoconciencia es decir, superar la conciencia. Hyppolite lo plantea en estos términos: "Ello no obstante, es esta separación del todo y de las partes, al mismo tiempo que su separación, lo que constituye la infinitud, la vida universal como <<vínculo del vínculo y del no vínculo $>>$ o como $<<$ identidad de la identidad y de la no identidad $>>$."18

Hemos finalizado la exposición de la dialéctica hegeliana que da vida a la idea de reconocimiento expuesta por Charles Taylor y ahora nos ocuparemos de analizar su texto ya con las herramientas conceptuales hegelianas presentes en la cual se fundamenta Taylor en su texto y que finalmente es lo que nos interesa para nuestra indagación.

\section{2-. TAYLOR Y LA NOCIÓN DE RECONOCIMIENTO E IDENTIDAD}

El ser humano en su afán de encontrar en el otro la respuesta idónea de su existir en el mundo mediante la comunicación intersubjetiva en todos los sentidos, ha conseguido su fin desde una perspectiva de reconocimiento e identidad como fundamental en su trayectoria hacia la definición de su modo de vida, que le permita sentirse realizado como ser humano, en tal sentido, Taylor nos presenta en su genial y también polémica interpretación los puntos

\footnotetext{
${ }^{16}$ Hegel, G.W.F., Escritos de Juventud..., cit., p. 401.

${ }^{17}$ Taylor, Ch., Argumentos filosóficos..., cit., p. 130.

${ }^{18}$ Hyppolite, J., Génesis y Estructura de la Fenomenología del Espíritu de Hegel, Madrid, Ediciones Península, 1974, p. 135.
} 
claves, neurálgicos para la comprensión de nuestro existir en el mundo en tanto que nuestra intersubjetividad.

¿Cuál es el trasfondo y pretensión de Taylor en la disposición de la comprensión en la categoría reconocimiento en el contexto actual? ¿A qué apunta la indagación de Taylor en cuanto a la obsolescencia de la estructura monológica ante la inevitable estructura dialógica? ¿La forma de Identidad está históricamente imbricada a la categoría reconocimiento, lo vemos ya en Hegel entonces cuál sería el mecanismo idóneo para exponerlas dentro de la tradición?

Responder a todas estas preguntas sugerentes es un verdadero reto, ahora bien, ¿qué es lo que cuestiona Taylor en su indagación? En primer lugar va contra el individualismo, en segundo lugar le inquieta la primacía de la pretensión de la Instrumental ratio referente a los fines axiológicos donde piensa que existe un menoscabo y en tercer lugar en el ámbito político-social el riesgo de la perdida de la "Libertad individual y colectiva".

En la introducción a "La ética de la autenticidad" Carlos Thiebaut dice que: "Taylor quiere encontrar en la idea de una política de $<<$ reconocimiento igual $>>$ la base de una reconceptualización de la esfera pública que atienda, a la vez, a las demandas de igualdad de las democracias modernas..."19 consideramos por tanto, que nuestro autor intenta explicar y organizar ese problema que subyace en nuestra contemporaneidad pero que también se subsume como cimiento en la política, en la democracia, en las repercusiones en el ámbito social, cultural, esto por supuesto tiene una trascendencia sustancial en los derechos fundamentales del hombre bajo el nombre de "Derechos Humanos" y además es causa inherente de preocupación en casi todos los países del mundo, de este modo conceptos como dignidad, los valores que están intrínsecamente conectados con el reconocimiento y por tanto en la identidad de cada individuo de una determinada sociedad y que recrea la intensidad en la cual nuestro autor muy preocupado por estos asuntos nos presenta a manera de ideas a través de sus textos.

Nos dice el filósofo canadiense que "El reconocimiento debido no es sólo una cortesía que debamos a la gente: es una necesidad humana vital" ${ }^{20}$ hemos de notar que la idea de reconocimiento tiene connotación importante con Identidad ¿Por qué? Hagamos una

\footnotetext{
${ }^{19}$ Taylor, Ch., La ética de la autenticidad, Barcelona, Editorial Paidós, S.A., 1994, p. 30.

${ }^{20}$ Taylor, Ch., Argumentos filosóficos..., cit., p. 294.
} 
descripción de manera que podamos ir aclarando este asunto, existe según Taylor una necesidad de reconocimiento, reconocimiento que debe ir de la mano con Identidad nuestro mundo, nuestra vida personal no sería posible si no tenemos contacto con el otro no podemos si quiera pensar en una posibilidad como esa, desde que nacemos entramos inmediatamente en contacto con el otro o lo otro en nuestro entorno y así comenzamos a percibir que existen cosas y escuchamos palabras etc., a medida que nos vamos desarrollando en esa medida vamos formando nuestros valores, nuestra cultura se hace inmanente a nuestro ser nos proyectamos de acuerdo a estos valores culturales que nos da forma, nos configura de acuerdo a una determinada sociedad es en ese sentido que no podemos ni pensar no estar en contacto con el otro, en nuestra opinión lo dicho anteriormente es identidad en cuanto reconocimiento del otro y viceversa.

Ahora bien, todo esto tiene implicaciones importantes en el hecho de ser reconocido bajo una identidad que nos va a permitir ser parte de un todo, Taylor nos habla en su texto "Argumentos filosóficos" que el ser humano al no ser reconocido -él lo llama "mal reconocimiento"- este ser humano se sentiría como un ser alienado de su propia existencia y en esa misma medida no tendría identidad, nos pone Taylor un ejemplo que nos parece excelente: "Algunas feministas han argumentado que las mujeres en las sociedades patriarcales han sido inducidas a adoptar una imagen desfavorable de sí mismas."21

Consideramos en lo siguiente la alienación de su ser "mujer" en tanto ser humano en términos excluyentes de esas sociedades patriarcales Taylor seguidamente nos da otro ejemplo importante en cuanto a las personas de color negro dice: "Una observación semejante se ha planteado en relación con los negros: la sociedad blanca ha proyectado durante generaciones una imagen degradante de ellos mismos, imagen que algunos de ellos no pudieron dejar de adoptar." 22 En Estados Unidos y lo mismo sucedió en Sudáfrica con la política de apartheid* a estas personas de color le han inculcado o le han hecho creer que son inferiores que son personas miserables y por tanto no tienen nada que buscar en una sociedad donde los blancos son los que dictan las pautas. Esto lo llama Taylor falso reconocimiento "el falso reconocimiento muestra no sólo la falta del debido respeto: puede infligir una herida

\footnotetext{
${ }^{21}$ Ibid., p. 293.

22 Ídem.

*Fue el resultado de una política de segregación racial impuesta en Sudáfrica por una minoría blanca y que se mantuvo casi todo el siglo XX hasta 1992 cuando se abolió el apartheid.
} 
dolorosa que causa en su víctima un mutilador autoodio."23 Pero no solo un autoodio también podría sin duda infligir un desprecio a la sociedad donde hace vida, en atención a lo expuesto observamos como en los EE.UU. donde el problema racial en el caso específico del negro y también entre otras culturas que hacen vida en ese país son degradados por los connacionales llámese blancos, etc. Eso es lo que se conoce como segregación racial y que está muy vigente aún hoy en pleno siglo XXI.

Algunos grupos humanos -llamados también minorías- son víctimas de estos abusos y descrédito se les imposibilita en primer lugar reconocerse como igual al otro, en segundo lugar lo que hace que este ser humano se sienta parte de una sociedad es decir asumiendo una identidad que lo configura como siendo él en el otro se ve anulado por este falso reconocimiento. Esto por su puesto trae implicaciones políticas, sociales que podría contribuir a un malestar en una sociedad que si lo vemos bien es mayoritaria, yendo por ejemplo a Sudáfrica donde una minoría de aproximadamente dos millones de blanco sometían mediante la política racista del apartheid a una cantidad considerable de negros, cerca de unos veintidós millones, dueños por cierto del espacio territorial donde han vivido por generaciones.

Estos ejemplos por supuesto nos aclara la importancia que tiene el ser reconocido en una determinada sociedad, el ser humano se siente incluido, se siente identificado y por tanto su dignidad está funcionando a plenitud. Cuando se da el reconocimiento inmediatamente la identidad funciona pues son inseparables.

Dentro de esta perspectiva, observamos que Dignidad es un concepto fundamental y evidentemente en esta coyuntura tiene su antecedente, Taylor lo explicará con detalle, sin embargo nos referiremos de inicio al concepto honor que tiene una aplicación fundamental pero restringida en su tiempo, el honor lo ostentaba solo algunos que lo merecían y por tanto se transformara como veremos más adelante en dignidad pero en un sentido universalista, más amplio. Taylor nos dice que:

El primero es el colapso de las jerarquías sociales, que solían ser la base del honor. Uso honor en el sentido del ancien régime, en que estaba intrínsecamente conectado con las desigualdades. En

\footnotetext{
${ }^{23}$ Ibíd., p. 294.
} 
este sentido, para que algunos tuvieran honores era esencial que no todos los tuvieran. ${ }^{24}$

La idea de honor ha servido para enterarnos y comprender en tiempos de Montesquieu cómo se le daba relevancia a un pequeño o reducido grupo privilegiado y además sólo algunos podían gozar de este honor "Así es que Montesquieu lo usa en su descripción de la monarquía: el honor es intrínsecamente una cuestión de preferencias." ${ }^{25}$ Luego de haber sufrido en el tiempo una transformación sustancial lo consideramos esencial porque dará paso a una noción contemporánea que estará en casi todas las cartas magnas del mundo como es la noción de Dignidad.

Ahora veremos cuál es su importancia y como lo desarrolla Taylor o mejor dicho como lo aplica a su texto, nuestro autor nos dice lo siguiente: "Frente a esta noción de honor, tenemos la moderna noción de dignidad, actualmente usada en un sentido universalista e igualitario, con la que nos referimos a la inherente $<$ dignidad de los seres humanos $>$ o a la dignidad ciudadana." 26 Esto que nos dice el autor canadiense es importante porque este concepto será la base esencial para la redacción de la Carta fundamental sobre los Derechos Humanos de la ONU (1948) de Paris.

Consideramos además, que la noción de Dignidad presenta una interesante connotación con lo que nos toca explicar respecto a reconocimiento e identidad y a su vez nos lleva a una implicación bien importante y que tiene que ver con la cultura democrática, eso indudablemente nos lleva nuevamente a pensar que el concepto dignidad con peso en nuestra investigación nos guiara y aclarara lo que plantea Taylor con referencia a reconocimiento e identidad.

Expliquemos un poco este asunto de cultura democrática enmarcado dentro de la noción de dignidad y sus inevitables implicaciones en el reconocimiento igualitario que nos habla Taylor, "La democracia ha conducido a una política de reconocimiento igualitario, que ha adquirido formas diversas a lo largo de los años y que ahora retorna en la forma de una exigencia de un igual estatuto para cultura y géneros."27 Qué quiere decir esto, la

\footnotetext{
24 ídem.

25 ídem.

26 lbíd., p. 295.

27 Ídem.
} 
compatibilidad con la idea de democracia del concepto dignidad permite de alguna manera confrontar aquellas diferencias en grupos culturales y al mismo tiempo grupos o géneros que se encuentran en situación de vulnerabilidad como por ejemplo las féminas que por cierto $-\mathrm{y}$ esto es indudable- han recuperado su dignidad mancillada en el pasado. Por ejemplo, hablemos ahora de ambos sexos 'El hecho de llamar a todos 'Mr.' 'Mrs.' En lugar de que algunos y algunas se les llame 'Lord' o 'Lady' y a los demás simplemente por su apellido..." 28 esto es fundamental tenerlo presente se ha dado un salto en la idea de igualdad en estos términos el reconocimiento del otro queda normalizado y esto es también válido para lo cultural.

A continuación entramos a considerar a la identidad individualizada ¿en qué consiste? Todos tenemos desde que éramos muy pequeños una configuración producto de los valores y costumbres trasmitidas por la familia y la comunidad donde nos hemos criado de esa manera y a la par logramos formar nuestra manera de ser, para actuar ante nuestra sociedad "Podemos hablar de una identidad individualizada, que es particularmente mía y que puedo descubrir en mí mismo. Tal noción surge junto con el ideal de ser fiel a mí mismo y a mi particular modo de ser." 29

Podemos por tanto pensar que subyace -y de hecho lo dice Taylor en su Ética de la autenticidad- en los problemas axiológicos que asoma a propósito del tema de la identidad individualizada, veamos a qué se refiere nuestros autor cuando dice que: "En la voz original, la voz interior tiene importancia porque nos dice qué es lo correcto a la hora de actuar."30 Creemos que si tomamos como fundamento esta voz interior que se transformará o reflejará en los valores que se ha ido formando podemos por tanto observar que la idea de Bien y del Mal se deriva de este reflejo de valores que se encuentran en los individuos de una determinada sociedad. "La noción de autenticidad se desarrolla a partir de un desplazamiento del acento moral según esta idea. En la perspectiva original, la voz interior es importante porque nos indica lo que es correcto hacer" ${ }^{\prime 31}$ en el desarrollo de nuestras vidas y que mediante los sentimientos morales podemos ir configurándonos en ser mejores personas con nosotros mismo -esto es importante- . Taylor seguidamente nos dice que "Existe un

\footnotetext{
28 ídem.

29 Ídem.

${ }^{30}$ Taylor, Ch., La ética de la autenticidad..., cit., p. 62.

${ }^{31}$ Taylor, Ch., Argumentos filosóficos..., cit., p. 296.
} 
determinado modo de ser humano que es mi modo; estoy llamado a vivir mi vida de esta manera, y no imitar la vida de ningún otro." 32 La autenticidad consiste que somos nosotros mismos con nuestras propias ideas, nuestra creatividad nuestro seguir en el mundo tal como somos sin convertir nuestro ser en una especie de mimesis sino en una constante poiesis personal. Pero existe siempre el peligro de ser influenciado por el otro y no escuchar esta voz interna sin embargo ese "principio de originalidad: cada una de nuestras voces tiene algo único que decir. No sólo no debería moldear mi vida según los requerimientos de conformidad externa; ni siquiera puedo encontrar fuera de mí mismo el modo según el cual vivir; sólo puedo hallarlo dentro de mí." 33 Ese ser sí mismo o más bien estar conmigo mismo en cuanto solo, de esta manera, puedo lograr hacerme exteriorizar mis potencialidades propias, es dentro de este marco a lo que indudablemente se refiere Taylor con la noción de autenticidad que según él es un modo de ser del ser humano en la contemporaneidad.

¿Cuál sería entonces la estrecha relación entre reconocimiento e identidad expuesta por Taylor? Él introduce otro problema fundamental, se trata del lenguaje, problema que preocupa en gran medida a casi todos los filósofos y sociólogos, pero aquí analizaremos puntualmente a Taylor en este sentido "Para mis propósitos quiero, tomar el término lenguaje en su sentido amplio, que otros modos de expresión por medio de los cuales nos definimos y que incluyen los lenguajes del arte, del gesto, del amor, etc." ${ }^{34}$ Observamos que una vez que logramos entender el problema de la autenticidad Taylor nos habla de cómo podemos aprender con el otro en una alteridad fundamental que permite formarnos dentro de una determinada sociedad, ya Hegel lo había visto de esa manera, está muy bien ese YO = YO es decir, somos iguales a nosotros mismo pero debe haber una negación de ese Yo que me permita saber y además agregaría, aprender del otro.

¿Pero cómo podemos hacer todas estas cosas? el lenguaje nos dice Taylor es por excelencia nuestra manera de comunicarnos y a la vez aprender del otro, él introduce dos conceptos que a nuestro criterio son importantes y que serviría para trazar nuestra investigación, la noción: monológico y dialógico, ¿en qué consiste estas dos nociones? nos

\footnotetext{
32 Ídem.

${ }^{33}$ Ibíd., p. 298.

${ }^{34}$ Ibid., p. 299.
} 
dice que "Este rasgo decisivo de la vida humana es su carácter fundamentalmente dialógico" "35 Hegel estaba en lo cierto cuando se refería a la lucha por el reconocimiento en tanto que sí y solo sí existe una intersubjetividad entre los seres humanos, pero también el hecho de que nos comprendamos nosotros mismos en esa medida estaremos conformes con el reconocernos en el otro y al mismo tiempo habiendo respeto al ser humano dentro de una determinada sociedad, la identidad como tal permite por tanto que exista una plenitud existencial, explicar esto no es fácil dada la complejidad del asunto, volvamos nuevamente a la cuestión que nos concierne a lo Dialógico, el lenguaje pero no sólo el lenguaje hablado sino también el lenguaje gestual, símbolos entre otros que nos sirve para intercambiar nuestros puntos de vista e intercambiar ideas con el otro, esto es bien importante para Taylor que se atreve a decir:

Nos convertimos en agentes humanos plenos, capaces de comprendernos a nosotros mismos y por tanto de definir nuestra identidad, a través de nuestra adquisición de ricos lenguajes expresivos humanos. -más adelante dice- Definimos nuestra identidad siempre en dialogo con, -a veces en lucha contra- lo que nuestros otros significativos quieren ver en nosotros. ${ }^{36}$

Entendiendo por significativos aquello a lo cual se refiere como agente externo en términos de igualdad es decir al otro (persona) que está ahí en frente, esto es en realidad esencial "En este sentido, la génesis de la mente humana no es monológica - no es algo que cada persona logra por sí misma -, sino dialógica." 37 Dialógica en el sentido de la comunicación en cualquiera de sus manifestaciones y que logra de alguna manera configurar al individuo en una constante intersubjetividad, es cierto que estamos influenciados de aquella fase monológica que nos vio nacer pero también es cierto que en el devenir de nuestras vidas entramos en una fase que es esencial en el ser humano, en una profunda alteridad ¿en qué sentido? el lenguaje en cualquiera de sus manifestaciones.

Por último entramos a considerar la identidad como noción fundamental, que por tanto como habíamos hablado más arriba no puede ir separada del reconocimiento, Taylor nos dice que "Considérese lo que entendemos por identidad. Quiere decir quiénes somos, $<$ de dónde venimos>. Porque éste es el sustrato contra el cual nuestros gustos, deseos, opiniones y

\footnotetext{
35 Ídem.

${ }^{36}$ Ídem.

${ }^{37}$ Ídem.
} 
aspiraciones adquieren sentido." 38 Esta definición que nos da el pensador canadiense permite que podamos entender por qué el concepto de identidad es fundamental y va necesariamente acompañado del concepto reconocimiento estas cualidades a las que se refiere en la cita que precede no obstante, nos permite entender que es de esta manera que la persona se siente reconocida e identificada consigo misma y con la sociedad en la cual está inmersa, sin embargo Taylor a propósito de lo que hemos hablado nos dice, que: “...el hecho de que yo descubra mi propia identidad no significa que lo haga aisladamente, sino que lo he negociado a través del diálogo, en parte abierto, en parte interno, con los otros." ${ }^{39}$ El reconocimiento sólo se logra en diálogo con el otro y por tanto se llega al reconocimiento igualitario "no es solo el modo apropiado para una sociedad democrática sana." $40 \mathrm{Si}$ este reconocimiento igualitario no se da es posible que cause traumas, problemas en las personas que pertenecen a una determinada sociedad o grupo social, esto es importante, es axial en Taylor, él introduce lo que llama esfera pública y que tendrá implicaciones en el llamado reconocimiento igualitario, diferenciándolo de la esfera íntima que como ya vimos más arriba es el diálogo con otros significativos.

La política de la dignidad igualitaria "se basa en la idea de que todos los humanos son igualmente dignos de respeto." ${ }^{41}$ en términos de la esfera pública tiene implicaciones sociales y políticos que subyace en la declaración de los derechos fundamentales del hombre conocido como Derechos Humanos y que tanto los organismos internacionales (ONU) entre otros tienen como fundamento para la organización política de casi todos los Estados del mundo, en este sentido Venezuela tiene plasmada esta idea de dignidad en su Carta Magna, el desconocimiento de esta noción de dignidad la cual hemos estudiado a la luz del pensamiento de Taylor podría causar daños irreparables en el individuo "La sola consideración -nos dice Taylor- de esta posibilidad representa ya una negación de la igualdad humana."42

Esto finalmente tiene implicaciones sobre un tema que para el pensador canadiense es fundamental el multiculturalismo que en definitiva abrió una discusión bien importante con algunos autores como Amy Gutmann, Susan Wolf, Steven Rockefeller y Michael Walzer

\footnotetext{
${ }^{38}$ Ibíd., p. 300.

${ }^{39}$ Ibíd., p. 301.

${ }^{40}$ Ibíd., p.303.

${ }^{41}$ Ibid., p. 307.

42 Ibíd., p. 308.
} 
entre otros introduciendo la idea de liberalismo como trasfondo y cuáles son las consideraciones que permite que las culturas sientan fuertemente estas ideas que hemos revisado sobre el reconocimiento e identidad. "Se considera -nos dice Taylor- que las sociedades liberales occidentales son fundamentalmente culpables con respecto a la imposición, en parte a causa de su pasado colonial y en parte por la marginación a que someten a segmentos de su población que provienen de otras culturas." ${ }^{\text {43 }}$ La preocupación de Taylor apunta sustancialmente al problema del "multiculturalismo" tanto es así que le dedicó el capítulo 12 de su "Argumentos filosóficos" a este problema y además propició una discusión un debate importante entre algunos autores que ya nombramos más arriba sobre dicho problema. Finalmente nos dice expresando preocupación por todo lo expuesto en su texto que: "La premisa de fondo de estas peticiones es que el reconocimiento forja identidad, especialmente en su aplicación fanonista: los grupos dominantes tienden a afirmar su hegemonía inculcando una imagen de inferioridad en los subyugados." ${ }^{44} \mathrm{Y}$ en eso consiste la lucha por el reconocimiento del que nos ha hablado Hegel y que tuvo una profunda influencia para Taylor.

No nos hemos referido y profundizado aquí a las críticas al argumento esgrimido por Taylor respecto a sus argumentos políticos en su texto, más bien hemos dedicado estas líneas al problema del reconocimiento e identidad como tal, será en otra investigación donde podamos incluir estas inquietudes que no dejan de ser importantes para una investigación futura. Es cierto que esta investigación no agota la inmensa literatura filosófica que existe sobre lo que hemos dicho aquí, sin embargo esperamos sirva éste como un abreboca para incentivar la investigación sobre Charles Taylor.

\section{BIBLIOGRAFÍA}

43 Ibid., p. 326.

${ }^{44}$ Ibíd., p 328. 
Fukuyama, Francis, El fin de la historia y el último hombre, Trad. P. Elías, Barcelona, Editorial Planeta, S.A., 1992.

Hegel, G.W.F., Escritos de Juventud, Trad. Zoltan Szankay y José María Ripalda, México, Editorial Fondo de Cultura Económico, 1998.

Hegel, G.W.F., Fenomenología del Espíritu, Trad. Wenceslao Roces, México, Editorial Fondo de Cultura Económica, 2006.

Hegel, G.W.F., Diferencia entre los sistemas de filosofia de Fichte y Schelling. Trad. M. ${ }^{a}$ Del Carmen Paredes Martín, Madrid, Editorial-Tecnos, S.A., 1990.

Heidegger, Martín. La Fenomenología del Espíritu de Hegel. Trad. Manuel E. Vásquez y Klaus Wrehde, Madrid, Editorial Alianza, S.A.1995.

Hyppolite, Jean, Génesis y Estructura de la Fenomenología del Espíritu de Hegel, Trad. Francisco Fernández Buey, Madrid, Ediciones Penisula, 1974.

Marcuse, Herbert, Razón y Revolución, Trad. Julieta Fombona de Sucre, Madrid, Alianza Editorial, S.A., 1976.

Nancy, Jean-Luc, Hegel. La inquietud de lo negativo, Trad. Juan Manuel Garrido, Madrid, Arena Libros, S.L. 2005.

Palmier, Jean, (2006). Hegel. Trad. Juan José Utrilla, México, Editorial Fondo de Cultura Económica, 2006.

Taylor, Charles, Argumentos filosóficos, Trad. Fina Birulés Bertrán, Barcelona, Editorial Paidós, 1997.

Taylor, Charles, Hegel. Trad. Francisco Castro, Carlos Mendiola y Pablo Lazo, Barcelona, Editorial Anthropos, S.A., 2010.

Taylor, Charles, La ética de la autenticidad, Trad. Pablo Carbajosa Pérez, Barcelona, Editorial Paidós, S.A., 1994.

Vásquez, Eduardo. Hegel un desconocido. ULA. Consejo de publicaciones. MéridaVenezuela, 1998 


\section{Luis Enrique Silva Viera. Currículo}

Luis Enrique Silva Viera. Profesor de Filosofía en la Pontificia Universidad Católica Santa Rosa. Caracas-Venezuela.

E-mails: novalisucv@gmail.com / filosofiaucv@gmail.com

Licenciado en Filosofía (2009), Licenciado en Educación: Mención Filosofía (2012), MSc. En Filosofía: Mención Filosofía y Ciencias Humanas (2019) Todos los estudios realizados en la Universidad Central de Venezuela. Actualmente realiza estudios de Maestría en Teología Dogmática por la Pontificia Universidad Católica Santa Rosa Caracas-Venezuela. Ponencias: Disertación sobre Karl Marx: La selva, La alienación del hombre, una interpretación. Disertación sobre Hegel: "La unidad dialéctica en la conciencia. La certeza Sensible en la Fenomenología del Espíritu de Hegel." Disertación: La filosofía de la reflexión en Hegel una aproximación a "Fe y Saber". Disertación: Charles Taylor: La noción de reconocimiento e identidad.

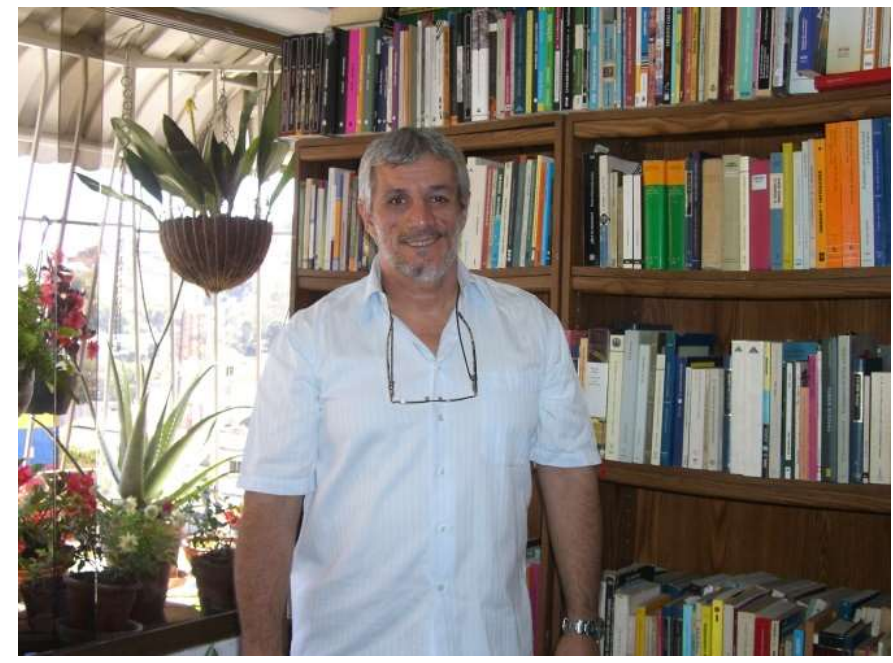

